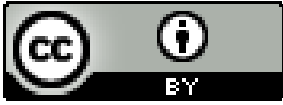

\title{
PERSPECTIVAS MULTICULTURAIS PARA O CURRÍCULO DE FORMAÇÃO DOCENTE ANTIRRACISTA
}

Ana Ivenicki ${ }^{1}$

Resumo: O presente artigo pretende contribuir sobre reflexões a respeito do currículo para o desafio ao preconceito com relação a identidades étnico-raciais marginalizadas. O argumento que apresento é que o currículo poderia se beneficiar de uma perspectiva multicultural pós/decolonial, com aportes do olhar interseccional e híbrido, que propõe o desafio à essencialização de identidades e a binarismos que as congelam. Desta forma, discuto implicações da abordagem multicultural para um currículo desafiador do racismo, propondo dimensões para a análise de experiências curriculares multiculturais, nesta perspectiva. Pretendo que o texto possa contribuir para reflexões e diálogos sobre caminhos futuros para perspectivas antirracistas e multiculturais no currículo e na formação de professores.

Palavras-chave: currículo, multiculturalismo, formação de professores, identidades étnicoraciais, antirracismo

\section{MULTICULTURAL PERSPECTIVES FOR AN ANTI-RACIST TEACHER EDUCATION CURRICULUM}

\begin{abstract}
The present paper intends to contribute for reflections about the curriculum that challenges prejudices related to marginalised racial and ethnic identities. The arguet is that the curriculum could benefit from a post/decolonial multicultural perspective that focuses on interseccional and hybrid approaches geared towards challenging the essentialisation of identities and binary approaches that freeze them. I discuss consequences of the multicultural approach for a curriculum that intends to challenge racism. I present possible dimensions for the analysis of multicultural curricular experiences, informed by that perspective. I intend the text to possibly contribute for raising reflections and dialogues about future roads towards anti-racist and multicultural curricular approaches for teacher education.
\end{abstract}

Key words: curriculum, multiculturalismo, teacher education, ethnic and racial identities, antiracism.

\section{PERSPECTIVAS MULTICULTURALES PARA EL PLAN DE ESTUDIOS DE FORMACIÓN DOCENTE ANTIRRACISTA}

Resumen: Este artículo tiene la intención de contribuir en las reflexiones sobre el plan de estudios al desafío del prejuicio con respecto a las identidades étnico-raciales marginadas. El argumento que hago es que el plan de estudios podría beneficiarse de una perspectiva multicultural post / decolonial, con contribuciones de la mirada interseccional e híbrida, lo que plantea el desafío de la esencialización de las identidades y los binarismos que las congelan. Por lo tanto, analizo las

\footnotetext{
1 Professora Emérita, Universidade Federal do Rio de Janeiro - UFRJ, Faculdade de Educação,
} Pesquisadora 1A do CNPq. E-mail: aivenicki@gmail.com 
implicaciones del enfoque multicultural para un plan de estudios que desafía el racismo, proponiendo dimensiones para el análisis de las experiencias de planes de estudios multiculturales, en esta perspectiva. Quiero que el texto contribuya a reflexiones y diálogos sobre futuros caminos para perspectivas antirracistas y multiculturales en el currículo y la formación de docentes.

Palabra clave: currículum, multiculturalismo, formación docente, identidades étnico-raciales, antirracismo

\section{PERSPECTIVES MULTICULTURELLES POUR LE PROGRAMME DE FORMATION DES ENSEIGNANTS ANTIRACISTES}

Résumé: Cet article vise à contribuer aux réflexions sur le curriculum pour remettre en question les préjugés liés aux identités ethniques et raciales marginalisées. L'argument que je présente est que le programme pourrait bénéficier d'une perspective multiculturelle post-décoloniale, avec des contributions du regard intersectionnel et hybride, ce qui pose le défi de l'essentialisation des identités et des binarismes qui les gèlent. De cette manière, je discute des implications de l'approche multiculturelle pour un programme de défi au racisme, proposant des dimensions pour l'analyse des expériences curriculaires multiculturelles, dans cette perspective. J'ai l'intention que le texte puisse contribuer aux réflexions et aux dialogues sur les voies futures des perspectives antiracistes et multiculturelles dans le curriculum et dans la formation des enseignants.

Mots clés: curriculum, multiculturalisme, formation des enseignants, identités ethnoraciales, antiracisme.

\section{INTRODUÇÃO}

O presente artigo parte da necessidade de se reconhecer a crescente relevância do currículo na formação das identidades docentes, numa perspectiva que encara a formação inicial e continuada como um continuum de desenvolvimento profissional, valorizando o papel central de experiências curriculares locais e internacionais que articulam o currículo à diversidade cultural, a perspectivas antirracistas e ao desafio ao silenciamento de identidades plurais. Reconhece que, embora a produção do conhecimento curricular esteja avançada, poderia se beneficiar de pesquisas que busquem experiências curriculares brasileiras e internacionais na formação de professores, analisando formas plurais de se conceber a internacionalização curricular no contexto de sociedades cada vez mais multiculturais.

Neste sentido, o artigo pretende contribuir sobre reflexões a respeito do currículo para o desafio ao preconceito com relação a identidades étnico-raciais marginalizadas. $\mathrm{O}$ 
argumento que apresento é que o currículo poderia se beneficiar de uma perspectiva multicultural pós/decolonial, com aportes do olhar interseccional e híbrido, que propõe o desafio à essencialização de identidades e a binarismos que as congelam. Desta forma, discuto implicações da abordagem multicultural para um currículo desafiador do racismo, propondo dimensões para a análise de experiências curriculares multiculturais, nesta perspectiva. Pretendo que o texto possa contribuir para reflexões e diálogos sobre caminhos futuros para perspectivas antirracistas e multiculturais no currículo e na formação de professores.

\section{MULTICULTURALISMO E FORMAÇÃO DOCENTE ANTIRRACISTA:}

\section{ABORDAGENS}

Pensar no currículo no âmbito do ensino superior e, mais especificamente, na formação de professores, implica em reconhecer seu impacto na formação das identidades docentes, enfatizando-se seu potencial para promover a valorização da diversidade cultural e desafiar o racismo, as desigualdades, os preconceitos e silenciamentos de vozes de grupos subalternizados, em função de raça, etnia, gênero e outros marcadores identitários (Banks, 2004; Candau, 2016; Ivenicki, 2015; Ivenicki \& Xavier, 2017; Ivenicki, 2018; Janoário, 2018; Lopes, Oliveira \& Oliveira, 2018; Macedo, 2019; Santiago \& Canen, 2013; Warren \& Canen, 2012). Nesta perspectiva, buscam-se possibilidades de diálogo e fertilização em um horizonte de currículo concebido multiculturalmente como espaço de diferenciação e de valorização da alteridade (Ivenicki, 2018; Macedo, 2016; Moreira \& Ramos, 2016; Miller, 2019).

Ao mesmo tempo, autores tais como Vargas \& Sanhueza, (2018) apontam ser importante desenvolver pesquisas que busquem aprofundar estratégias curriculares de gestão e atenção à diversidade que possam ir além das práticas de reconhecimento das mesmas, mas que envolvam uma problematização crítica das desigualdades que se legitimam e se reproduzem nos espaços educativos, por meio de modelos multiculturalistas de formação docente que superem o preparo exclusivo em competências técnicas pertinentes a áreas de estudo.

Neste sentido, Ranniery e Macedo (2018) salientam que as ações cotidianas, escorregadias, invisíveis, têm se constituído em novos modos de vida política, em espaços 
que abrem escolas e currículos à diferença, representando o que denominam de " cenas curriculares", em contraposição a efeitos universalizantes e, muitas vezes, homogeneizadores, de políticas mais amplas curriculares. Nesta mesma visão, Grimmett \& Halvorson (2010) propõem que a pesquisa na área de Currículo poderia se beneficiar de estudos voltados a formas concretas emancipatórias de desenvolvimento curricular. Stein (2017), em perspectiva semelhante, argumenta que é rara a produção do conhecimento sobre experiências de internacionalização do currículo no ensino superior em perspectivas desafiadoras de visões colonialistas que legitimam "efeitos repressivos, disciplinadores e biopoliticamente reprodutivos de desigualdades" (tradução livre, p. 531). Autores como Ball (2012) recomendam, ainda, a necessidade de pesquisas que sejam generativas, termo definido pela referida autora como expressando uma perspectiva de protagonismo para a construção de alternativas concretas de currículos multiculturais, para além de estudos teóricos sobre o assunto.

Nesta perspectiva, argumentamos que o olhar multicultural pode representar contribuição relevante na discussão sobre as relações étnico-raciais e as perspectivas antirracistas no currículo e na formação de professores.

Multiculturalismo é geralmente compreendido como um conjunto de estratégias que articulam teoria e ações práticas para a melhoria das vidas de grupos marginalizados (Candau, 2016; Ivenicki, 2018; Janoário, 2018). Tomando a categoria identidade como central, o multiculturalismo valoriza a pluralidade de identidades individuais (de todos os sujeitos), coletivas (agrupadas em torno de marcadores-mestre, tais como raça e etnia) e institucionais (representando o clima institucional, sendo importante que se constituam como espaços democráticos de valorização da diversidade).

É importante sinalizar que o multiculturalismo contempla um espectro que vai de abordagens mais liberais e folclóricas, nas quais se valorizam ritos, datas comemorativas e outros aspectos das identidades plurais, até perspectivas mais críticas e pós-coloniais, que enfatizam a necessidade de se desafiar preconceitos e relações de poder desiguais que prejudicam identidades sexuais, de gênero, étnico-raciais e outros grupos marginalizados, defendendo hibridizações e problematizando a construção discursiva dos estereótipos (Ivenicki, 2018; Ivenicki \& Xavier, 2015; Warren \& Canen, 2012) e a fixação de discursos identitários em políticas curriculares (Macedo \& Lopes, 2009). 


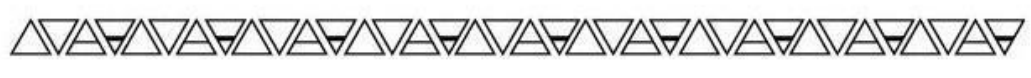

É relevante destacar que processos de colonização e políticas contemporâneas que ainda se imbuem dessas perspectivas colonialistas operam em termos de controle e negação do pluralismo. Marques (2018) ilustra essa ideia, ao discorrer sobre o fato de que, mesmo após a abolição da escravidão, a herança de um passado colonialista e escravista tem marcado as relações sociais em nossa sociedade. Tal processo tem resultado, de um lado, na construção da identidade coletiva negra em meio a conflitos e preconceitos. De outro, porém, tem resultado em movimentos de resistência com a ressignificação positiva desta mesma identidade, conforme ilustrado, pela referida autora, no contexto do acesso ao ensino superior e das oportunidades ali desenvolvidas.

Neste horizonte, enfatiza-se a importância do reconhecimento de perspectivas decolonais nas pedagogias críticas multiculturais. Tais perspectivas multiculturais decoloniais voltam-se à problematização de currículos baseados em perspectivas hegemônicas colonialistas, com o intuito de promover a valorização dos saberes nativos de grupos subalternizados. Desta forma, buscam fortalecer suas identidades individuais e coletivas em espaços em que a identidade institucional seja coletivamente construída de modo a que favoreça, cada vez mais, a diversidade, o antirracismo e a equidade social.

\section{DIMENSÕES DO CURRÍCULO MULTICULTURAL E IDENTIDADES ÉTNICO-RACIAIS}

Argumentamos que pensar em experiências curriculares multiculturalmente orientadas passa por duas perspectivas de análise: a polissemia do conceito de multiculturalismo, brevemente discutida na seção anterior; e as dimensões das experiências curriculares analisadas.

Em termos das dimensões focalizadas nas experiências curriculares, uma delas refere-se às formas pelas quais as identidades coletivas plurais são trazidas, seja por meio de suas narrativas e estratégias de resiliência e empoderamento, e/ou pela articulação dessa dimensão à própria produção de conhecimento em áreas educacionais e pedagógicas (incluindo temas e estratégias de aprendizagem a serem desenvolvidas).

Lopes, Oliveira \& Oliveira (2018), por exemplo, enfatizam que as questões relacionadas às diferenças estão na escola, assim como permeiam as próprias estruturas das políticas curriculares e dos discursos pedagógicos, disciplinares, midiáticos, políticos, 
sociais, religiosos e familiares, sendo o currículo espaço privilegiado de construção, de reprodução ou de tensionamento, resistência e deslocamento de modelos dominantes que silenciam identidades e perpetuam desigualdades. Ainda nesta dimensão, Maciel \& Garcia (2018), desenvolveram pesquisa a partir de histórias orais de professoras lésbicas, analisando como o meio discursivo/cultural dos discursos da lesbianidade — nos quais a estabilidade da estrutura binária do sexo é subvertida, as categorias das identidades são abertas e a política da identidade sexual é compreendida como produção política e cultural — possibilita o protagonismo de experiências e pedagogias próprias dessas professoras, com implicações curriculares.

Ao mesmo tempo, as experiências curriculares que se desenvolvem a partir de temáticas multiculturalmente orientadas, focalizadas sobre histórias de vida e perspectivas de identidades culturais marginalizadas, tais como identidades étnicoraciais, podem ser imbricadas com o que temos denominado de temáticas mais convencionais ligadas a áreas curriculares escolares e pedagógicas, na formação docente (Ivenicki, 2018). Isto porque, para além do foco sobre identidades, ilustrado anteriormente, a questão do conhecimento é outro aspecto relevante na área curricular. Neste caso, experiências curriculares narradas, com sensibilidades multiculturais, voltamse a formas pelas quais o conhecimento é "multiculturalizado", como indicado por pesquisas desenvolvidas, por exemplo, por autores como Santiago \& Ivenicki (2017) e Silva Júnior e Ivenicki (2019) que ilustram, respectivamente, o currículo de Artes e o currículo de Música a partir de estratégias de desenvolvimento de perspectivas desafiadoras de noções heteronormativas e hegemônicas na dança e na música, respectivamente.

Neste horizonte, que enfatiza o conhecimento multiculturalizado, Boaler \& Sengupta-Irving (2012) trazem tal dimensão de experiências curriculares multiculturais para o contexto do ensino de matemática, disciplina normalmente associada com perspectivas "universalistas" e desprovida de gênero. Os referidos autores mostram que a forma tradicional de ensino da matemática, por meio de demonstrações e, em seguida, por meio de prática e memorização, acaba por perpetuar o fracasso de identidades de gênero plurais, que não se veem representadas em tal método. Além do mais, as evidências de pesquisas citadas por Boaler \& Sengupta-Irving (2012) demonstram que tal forma de apresentação da matemática de modo abstrato e descontextualizado acaba por 
ser mais "alienadora" para identidades femininas do que masculinas, por exemplo e para grupos culturais minoritários, perpetuando a desigualdade de gênero, particularmente nas séries mais avançadas. Os referidos autores propõem, pois, que o conhecimento matemático seja trabalhado como disciplina discursiva, contextualizada, promovendo diálogos, questionamentos e discussões.

Também, trabalhos anteriores (Ivenicki \& Xavier, 2017) ilustram a articulação da perspectiva multicultural aos conhecimentos pedagógicos da formação de professores em projetos de extensão em parceria com secretarias de educação, nos quais oficinas desenvolvidas com professores de redes públicas de ensino resultaram em conhecimentos pedagógicos (tais como nas áreas de avaliação e de construção de projetos políticopedagógicos, por exemplo) ancorados na pluralidade cultural, bem como em processos de construção curricular em que os conteúdos curriculares escolares foram imbuídos de sensibilidades multiculturais, desafiando preconceitos contra identidades coletivas plurais, incluindo étnico-raciais.

Nesta perspectiva de conhecimento pedagógico na formação docente, Candau (2016) sugere que a Didática, ao passar por uma reconfiguração, tomando a perspectiva multi/intercultural crítica como eixo fundamental, pode romper com processos educativos homogeneizadores. Candau (2016) defende que o multiculturalismo e a interculturalidade representam categorias que, ainda que polissêmicas, têm trazido contribuições para se pensar na construção de escolas e currículos que favoreçam a expressão das matrizes culturais plurais. Ao mesmo tempo, concordamos com a referida autora quando aponta, em perspectivas críticas, que o currículo, na perspectiva multicultural, não desvincula as questões das diferenças e das desigualdades, reconhecendo que estas são atravessadas por conflitos de poder, preconceitos e discriminações com relação a identidades de gênero, raça, etnia, orientação sexual e outras, invisibilizadas em cotidianos educacionais.

Stein (2017), por sua vez, propõe que perspectivas multiculturais curriculares com "thin inclusion" (p. S32), ou seja, com "fraca inclusão" (nossa tradução livre), promovem, apenas, a incorporação de textos e perspectivas de valorização da diversidade cultural em datas e itens curriculares específicos. A referida autora exemplifica este olhar com esforços curriculares de se designar um dia ou uma semana de cursos de formação docente para se discutir raça ou gênero, deixando que o restante da temática curricular, no referido 
curso, silencie a respeito de questões relacionadas às desigualdades e preconceitos, como a racialização, o colonialismo, o patriarcalismo e a heteronormatividade.

Ainda que tal perspectiva de multiculturalismo com fraca inclusão curricular possa favorecer uma inicial conscientização sobre a pluralidade cultural, Stein (2017) aponta para a necessidade de incorporação de visões críticas pós-coloniais e decoloniais, que permitam o acesso problematizador a processos históricos e diferencialistas de poder acumulados nas formas pelas quais o conhecimento institucional hegemônico se perpetua, nos contextos educacionais, no âmbito do ensino superior. Tal visão mais crítica pode ser incentivada no que Stein (2017) denomina de perspectiva multicultural curricular de "thick inclusion" (p. S33), ou seja, de uma "forte inclusão" (nossa tradução livre). Nesta perspectiva, o currículo na formação docente e no âmbito do ensino superior estaria voltado ao incentivo a que estudantes problematizem constantemente as "boas intenções" (p. S34) do currículo e que incorporem tradições não ocidentais e não hegemônicas na abordagem curricular. Tal perspectiva crítica, entretanto, conforme Stein (2017), ainda desenvolve o multiculturalismo curricular no escopo do próprio currículo dominante, ainda que avance com relação à perspectiva anterior.

No escopo do currículo internacional, Stein (2017) aponta que essas visões multiculturalistas de fraca ou forte inclusão curricular têm predominado, juntamente com outras que preconizam interdisciplinaridade e instituições alternativas, que a referida autora aponta como pouco favorecedoras de formas emancipatórias de promoção de epistemes alternativas a visões hegemônicas do currículo. Sugere, nesta linha de argumentação, visões pós-coloniais e decoloniais em uma abordagem por ela denominada de "ecology of knowledges approach" (p. S42), ou seja, "ecologia dos saberes" (nossa tradução livre). Tal visão seria constituída do desafio à universalidade preconizada pelo pensamento ocidental, articulando, à crítica a este pseudo-universalismo, o estudo dos saberes deslegitimizados pelas perspectivas colonialistas. Nesta perspectiva, Stein (2017) defende que um leque de conhecimentos pode coexistir em contextos curriculares específicos, sem que haja uma intenção de mostrar a habilidade que esses conhecimentos possuem de representar "objetivamente" a realidade, em todos os contextos. Nesta ecologia de saberes em visões pós e decoloniais, múltiplos saberes e conhecimentos não lutam por hegemonia curricular, pois cada um deles é parcial, provisório e ligado a contextos específicos, promovendo sempre problematizações e respostas "incompletas". 
Tal visão tem contribuído para o pensamento multicultural curricular e tem também sido apontada em perspectivas pós-fundacionais e pós-estruturalistas do currículo, como a defendida por autores tais como Miller (2019), que argumenta que o foco do currículo deveria ser no que ela denomina de " differing differences" (diferenciando as diferenças), ou seja: um processo constante de tradução cultural, que possibilita a que educadores e pesquisadores em educação possam compreender a relevância do confronto de perspectivas culturais de grupos plurais, incorporando, no currículo, múltiplas versões das identidades presentes nos espaços educacionais, incluindo saberes de grupos étnico-raciais plurais. Nesta perspectiva, também Macedo (2019), a partir de Bhabha (2013), sugere que o currículo seja pensado como um terceiro espaço de enunciação, defendendo que propicie a exposição a uma alteridade radical que não pode ser prevista, antecipada ou regulada.

\section{O OLHAR MULTICULTURAL HÍBRIDO E INTERSECCIONAL}

Chueh (2005) defende que "as novas tentativas de valorizar a diferença cultural têm sido prejudicadas, justamente, pela sua dependência ao conceito de oposição binária, assim como à sua incapacidade de transformar o sentido metafísico dessas oposições binárias" (p. 375).

No que diz respeito às identidades étnico-raciais, observa-se que a identidade coletiva negra, alvo de racismos e de processos xenofóbicos, tem interessantes análises desenvolvidas por autores tais como Munanga (2000), Oliveira (2006), D’Adesky (2001) e Siss (2003). Os referidos autores têm contribuído para o refinamento do conceito de identidade negra com relação à cultura africana e suas matrizes, enfatizando que perspectivas antirracistas deveriam levar em conta essas sensibilidades e contextualizações plurais, dadas as plurais proveniências geográficas e culturais da referida identidade.

A linguagem antirracista desafia construções discursivas dicotômicas e essencializadas, com relação à construção identitária e pode ser ilustrada, por exemplo, por McCarthy (2005), que sugere que a produção cultural deveria "anunciar e reconhecer as complexidades, os alcances e as imbricações que se dão nos contatos humanos, nas produtividades, nas subjetividades e nos encontros raciais entre diferentes indivíduos e 
grupos” (p. 416). O referido autor argumenta que a existência de processos de hibridização cultural resultam de traduções, reinterpretações e rearticulações de universos culturais e étnico-raciais, desafiando perspectivas xenofóbicas e posturas racistas que separam, em linhas rígidas, o eu e o outro, o indígena e o estrangeiro, o branco e o negro e assim por diante.

Tais fatores demonstram a complexidade, a variabilidade e a hibridização presentes na formação identitária. Se levados em consideração, evitam que pedagogias aantirracistas caiam na armadilha de simplesmente pensar em abordagens curriculares aditivas, que, além de serem ineficazes contra o preconceito e o racismo, em seus aspectos discursivos, também, acabam por focalizar as identidades marginalizadas de forma separada, ignorando as rearticulações e decodificações que levam às culturas e identidades híbridas.

As considerações acima apontam para a relevância da construção de pedagogias antirracistas que superem discursos congelados e que desnudem suas influências materiais e ideológicas, de modo a desestabilizá-los e a fornecer elementos que contribuam, cada vez mais, para propostas pedagógicas que levem em conta o contexto singular em que nossas identidades circulam.

A partir do exposto, os conceitos de hibridismo e interseccionalidade, no contexto em discussão, apresentam-se fecundos para a análise de narrativas, vozes e identidades locais, transitórias e instáveis presentes nas formas pelas quais o currículo passa a ser pensado e vivido, em experiências curriculares de formação docente em perspectivas multiculturais com sensibilidades críticas pós-coloniais e decoloniais.

Conforme Bhabha (2015), o conceito de hibridismo encontra-se no cerne de perspectivas pós-coloniais e decoloniais, referindo-se ao que ele denomina de empoderamento das formas fronteiriças de construção de identidades que podem ser assimétricas e contraditórias, por meio do planejamento de modos de incentivar seus agenciamentos e protagonismos, nas relações políticas de poder.

Estreitamente ligado ao hibridismo, o conceito de interseccionalidade tem sido igualmente empregado em perspectivas multiculturais. De acordo com Coleman (2019), a interseccionalidade foi popularizada inicialmente por Kimberlé Crenshaw que, em artigo escrito em 1991 denominado de "Mapping the Margins" (Mapeando as Margens), explicou como sujeitos apresentam-se como mulheres e também pessoas de cor, 
marginalizadas por discursos que, ainda que bem intencionados multiculturalmente, são estruturados para responder a um ou outro marcador de identidade, sem a necessária visão articulada de ambos- gênero e identidade étnico-racial. Tais considerações partem da ideia de que vivemos vidas complexas interseccionadas por sistemas de privilégio e opressão. Neste sentido, conforme Coleman (2019), uma mulher lésbica negra estaria sofrendo mais preconceito do que uma outra heterossexual, por exemplo.

Tal visão pode ser ilustrada também em artigos contidos em um volume publicado pelo Grupo de Trabalho (Special Interest Group - SIG) da American Educational Research Association (AERA) em 2016, que mostra preocupações com a identidade de gênero articulada à identidade étnico-racial, no contexto da formação de professores. Neste caso, autores falam do impacto de professoras do ensino superior negras (Ross, 2016), latinas (Stevensons, 2016) e asiáticas (He, 2016), em um contexto de ensino superior predominantemente branco. De forma geral, tais artigos apresentam histórias de vida das autoras, assim como ilustrações de como trabalham o currículo de modo a desafiar estruturas de opressão, discriminação e racismo. Apresentam dinâmicas que desenvolvem com os alunos, tais como a escolha de textos que tensionam binômios identitários e propõem o hibridismo como categoria central.

No Brasil, pesquisa desenvolvida por Almeida et. al. (2018) confirma tal perspectiva, sinalizando formas pelas quais as categorias raça e etnia se entrecruzam, muitas vezes, com outras configurações sociais, tais como classe, gênero e sexualidade, ressaltando a importância de se reconhecer tal imbricação para a formulação de estratégias de ensino e aprendizagem voltadas para a compreensão e o respeito à diversidade cultural e para pedagogias antirracistas. Também, estudo desenvolvido por Silva Júnior e Ivenicki (2019) articula as percepções que circulavam na escola sobre masculinidades e suas imbricações com as construções discursivas dos mesmos sujeitos sobre raça e etnia.

Também, é importante notar que o conceito de hibridismo tem sido empregado, ainda, na visualização do currículo como artefato híbrido propriamente dito. Isto significa, conforme autores como Moreira \& Ramos (2016), Lopes, Oliveira \& Oliveira (2018) e Macedo (2006), que o currículo constitui-se, ele próprio, em um híbrido cultural, um conjunto de discursos e narrativas que não apenas podem vir a reproduzir intenções 
políticas hegemônicas, mas que as traduzem em processos de reinterpretação plurais nos diversos contextos em que circulam.

No contexto do currículo, o reconhecimento dos conceitos de hibridismo e de interseccionalidade identitária significa o desafio a formas essencializadas de se conceber as identidades docentes e discentes, de modo que o currículo possa propiciar o reconhecimento das diferenças dentro das diferenças. Neste sentido, tais perspectivas podem vir a desafiar projeções homogeneizadoras e regulações identitárias, trazendo possibilidades de resistência e ressignificação positiva de identidades, incluindo as identidades étnico-raciais.

\section{CONCLUSÕES}

O presente artigo apresentou possibilidades de articulação de perspectivas multiculturais ao horizonte da formação docente valorizadora da pluralidade de identidades e antirracista. Argumentou que olhares pós-coloniais e decoloniais multiculturais sobre o currículo ampliam a visão curricular, uma vez que sugerem que, para além do foco sobre as desigualdades, trata-se de analisar a identidade como híbrida, transitória e fluida.

Neste sentido, passa-se a "desessencializar" a categoria identidade, o que, segundo esta perspectiva, poderia ajudar professores, futuros professores e alunos a compreenderem a relevância de se desafiar abordagens dicotômicas que acabam por congelar "eu" e o "outro". Ao contrário, estas abordagens preconizam que a diferença deve ser entendida como ligada a processos de colonização que subestimam os saberes do "colonizado". Tais processos, nessa visão, ainda seriam inerentes a nosso currículo e à própria formação de professores e, portanto, poderiam ser desafiados em perspectivas pedagógicas de experiências curriculares multiculturalmente orientadas, com sensibilidades críticas, pós-coloniais e decoloniais. Em tais experiências, defendemos que o reconhecimento dos saberes nativos de identidades coletivas marginalizadas, como as identidades étnico-raciais plurais, pode se dar: em termos de suas contribuições e histórias de vida; e por intermédio da articulação dos saberes curriculares das diversas áreas ao olhar multicultural, valorizador de formas plurais de conceber a construção do conhecimento nessas mesmas áreas. 
Longe de esgotar a discussão sobre possibilidades curriculares para a formação de professores multicultural e antirracista, o artigo pretendeu levantar questões relativas às possíveis abordagens multiculturais no currículo antirracista, apontando para a relevância do reconhecimento da imbricação das identidades coletivas em processos de hibridização e interseccionalidade. Esperamos que o artigo possa contribuir para reflexões que ajudem o desenvolvimento de formas emancipatórias de promoção de epistemes alternativas a visões hegemônicas do currículo, promovendo a valorização da formação docente multicultural e antirracista, em um mundo cada vez mais diverso e plural.

\section{REFERÊNCIAS BIBLIOGRÁFICAS}

ALMEIDA, Neil Franco Pereira de; AMÂNCIO, Marcia Helena; SANTOS, Sérgio Pereira dos; SALES, Leydiane Vitória. Formação Docente e a Temática Étnico-Racial na Revista Brasileira de Educação da ANPEd (1995 - 2015). Revista Brasileira de Educação, v. 23, e230033, 2018.

ALVES, Nilda e OLIVEIRA, Inês Barbosa de. Uma História da Contribuição dos Estudos do Cotidiano Escolar no Campo do Currículo. In: Lopes, Alice Casimiro e Macedo, Elizabeth (orgs.), Currículo: debates contemporâneos. São Paulo: Cortez Editora, 2002, p. 78 - 102.

AYSCUE, Jennifer B. \& SIEGEL-HAWLEY, Genevieve. Magnets and School Turnarounds: Revisiting Policies for Promoting Equitable, Diverse Schools. Education Policy Analysis Archives, v. 27 n. 72, online, file:///C:/Users/aiven/Documents/4248-18962-1-PB.pdf, 2019.

BALL, Arnetha. To Know is not Enough: knowledge, power and the zone of generativity. Educational Researcher, v. 41, n.8, 2012, p. 283-293.

BANKS, James. Introduction: Democratic Citizenship Education in Multicultural Societies. In: Banks, J. A. (Ed.). Diversity and Citizenship Education. San Francisco: John Willey \& Sons, Inc., 2004, p.3-15.

BHABHA, Homi. Nuevas minorias, nuevos derechos: Notas sobre cosmopolitismos vernáculos. Buenos Aires: Siglo Veintiano Editores, 2013.

BHABHA, Homi. Foreword. In: Werner, Pina \& Modood, Tarqi (Eds.). Debating Cultural Hybridity: multicultural identities and the politics of anti-racism. London,

UK: Zed Books, 2015.

BOALER, Jo \& SENGUPTA-IRVING, Tesha. Gender Equity and Mathematics Education. In: Banks, James. (ed.), Encyclopedia of Diversity in Education. 1ed. New York: Sage Publications, v. 2, 2012, p. $972-975$. 
CANDAU, Vera Maria Ferrão. "Ideias-Força" do Pensamento de Boaventura Souza Santos e a Educação Intercultural. In: Candau, Vera Maria Ferrão (org.), Interculturalizar, Descolonizar, Democratizar: uma educação "outra”? Rio de Janeiro: 7 Letras, 2016, p. 76 - 96.

CHUEH, Ho-chia, The Multicultural Caveat: a pedagogy of the politics of difference. In: Canen, A. \& Peters, M. (Eds), Issues and Dilemmas of Multicultural Education: theories, policies and practices, Policy Futures in Education, v. 3, n. 4, 2005, p. 359 - 377.

COLEMAN, Arica. What's intersectionality? Let these scholars explain the theory and its history, Time: History and Feminism, https: time.com/intersectionality theory, online, acesso em 10 de junho de 2019, 2019.

D’ADESKY, Jacques. Pluralismo Étnico e Multiculturalismo: racismos e anti-racismos no Brasil. Rio de Janeiro: Ed. Pallas, 2001.

GRIMMETT, Peter e HALVORSON, Mark. From Understanding to Creating Curriculum: the case for the co-evolution of re-conceptualized design with re-conceptualized curriculum. Curriculum Inquiry, v. 40, n.2, 2010, p. $241-262$.

HE, MING FANG. Thriving in-between landscapes of education. Sophist's Bane: a journal of the society of Professors of Education, v. 8, n. 1, Washington, p. 48-51. Disponível em: <http://societyofprofessorsofeducation.com>. Acesso em: 20. fev 2017, 2016.

IVENICKI, Ana. Multiculturalismo e Formação de Professores: dimensões, possibilidades e desafios na contemporaneidade. Ensaio, Avaliação e Políticas Públicas em Educação, v. 26, 2018, p. $1151-1167$.

IVENICKI, Ana e XAVIER, Giseli Pereli de Moura Diversidade e Formação de Professores na Educação Básica: contribuições do multiculturalismo e da pesquisa-ação. In: Rios, Jane Adriana Vasconcelos. (org.). Diferenças e Desigualdades no Cotidiano da Educação Básica. 1ed.Campinas: Mercado das Letras, 2017, v. 1, p. 57-70.

JANOÁRIO, Ricardo. Diálogos Interculturais. Rio de Janeiro: Ed. Ayvu, 2018.

LOPES, Alice Casimiro; Oliveira, Anna Luiza A. R. de Martins de \& Oliveira, Gustavo Gilson Sousa de. Apresentação: gênero e sexualidade na educação brasileira- tensões, deslocamentos e horizontes. In: Lopes, Alice Casimiro; Oliveira, Anna Luiza A. R. de Martins de \& Oliveira, Gustavo Gilson Sousa de. Os Gêneros da Escola e o (im)possível silenciamento da diferença no currículo. Recife: Ed. UFPe, 2018, p. 7 - 20.

MACEDO, Elizabeth. Curriculum Beyond Normativity: difference concealment in Brazilian Curriculum Policies. In: Roundtable Differing Differences: transnational curriculum spaces and inquiries in post-truth times, Division B, Curriculum Studies, 2019 AERA Annual Meeting, 5-9 de Abril, Toronto, Canadá, 2019. 
MACIEL, Patricia Daniela e GARCIA, Maria Manuela Alves. Lesbianidade como Arte da Produção de Si e Suas Interfaces no Currículo. Revista Brasileira de Educação v. 23 e230022, 2018.

MARQUES, Eugenia Portela de Siqueira. O Acesso à Educação Superior e o Fortalecimento da Identidade Negra. Revista Brasileira de Educação, v. 23 e230098, 2018.

MCCARTHY, Cameron. English Rustic in Black Skin: post-colonial education, cultural hybridity and racial identity in the new century. In: Canen, Ana. \& Peters, Michael. (Eds), Issues and Dilemmas of Multicultural Education: theories, policies and practices, Policy Futures in Education, v. 3, n. 4, 2005, p. 413-422.

MILLER, Janet. Curricular Multiplicities and Constant Cultural Translations. In: Roundtable Differing Differences: transnational curriculum spaces and inquiries in post-truth times. Division B, Curriculum Studies, 2019 AERA ANNUAL MEETING, 5-9 de Abril, Toronto, Canadá, 2019.

MOREIRA, Antonio Flavio Barbosa e RAMOS, Rosane Karl. Mobilidade Educacional e a Internacionalização dos Estudos Curriculares. Revista Teias, v. 17, n.45, 2016, p. 163-175.

MUNANGA, Kabelengue. Uma Abordagem Conceitual das Noções de Raça, Racismo, Identidade e Etnia. In: Brandão, A. A. P. (Ed.). Cadernos PENESB- Programa de Educação sobre o Negro na Sociedade Brasileira, n. 5, 2000, p. 15 - 34.

OLIVEIRA, Iolanda. Educação e População Negra: especialistas em sala de aula e no contexto escolar. In: Oliveira, Iolanda. (ed.), Cadernos PENESB- Programa de Educação sobre o Negro na Sociedade Brasileira, n. 6, 2006, p. 179 - 208.

RANNIERY, Thiago. \& MACEDO, Elizabeth. Políticas do Vivível: diferença, teoria e democracia por vir. In: Lopes, A. C.; Oliveira, A. L. A. R. M. de; \& Oliveira, G. G. Sousa de (orgs.), Os Gêneros da Escola e o (im)possível silenciamento da diferença no currículo. Recife: Ed. UFPE, 2018, p. 21- 50.

ROSS, Sabrina. Dangerous terrain, reflections of a black woman teacher education working within predominantly white universities, Sophist's Bane: a journal of the society of professors of education, v. 8, n.1, Washington, p. 5-9, 2016. Disponível em: <http:// societyofprofessorsofeducation.com>, 2016. Acesso em: 20 fev. 2017.

SANTIAGO, Renan e IVENICKI, Ana. Diversidade Musical e Formação de Professores (as): qual música forma o (a) professor (a) de música? Revista Faeeba, v. 26, 2017, p. 187-204.

SILVA JÚNIOR, Paulo Melgaço e IVENICKI, Ana. Entre Sexualidades, Masculinidades e Raça: Contribuições do Multi/ Interculturalismo Para A Prática Pedagógica. Revista Tempos e Espaços Em Educação (Online), v. 12, 2019, p. 125-144.

STEIN, Sharon. Contesting Coloniality: The Persistent Challenges of Addressing Epistemic Dominance in Higher Education - considering the case of curriculum internationalization. Comparative Education Review, vol. 61, n. S1, 2017, p. S25 - S50. 
STEVENSON, Alma. Myself, my choice, my praxis: a Latina's journey into academia. Sophist's Bane: a journal of the society of Professors of Education, v. 8, n.1, Washington, p. 25-28, 2016. Disponível em:http://societyofprofessorsofeducation.com, 2016. Acesso em: 20 fev. 2017.

VARGAS, Felipe Jiménez e SANHUEZA, Carmen Montecinos. Diversidad, modelos de gestión y formación inicial docente: desafíos formativos desde una perspectiva de justicia social. Revista Brasileira de Educação, v. 23, e230005, 2018.

WARREN, Jonathan. \& CANEN, Ana. Racial Diversity and Education in Brazil. In: Banks, James. (Org.). Encyclopedia of Diversity in Education. 1ed. New York: Sage Publications, v. 1, 2012, p. 262-264.

Recebido 30/03/2020

Aprovado em 30/04/2020 